\title{
Thyroid Leiomyosarcoma: Primary or Metastasis? That's the Question!
}

\author{
Simonetta Piana - Riccardo Valli • Anna Maria Foscolo
}

Published online: 21 September 2011

(C) Springer Science+Business Media, LLC 2011

A 59-year-old woman presented with a rapidly expanding anterior neck mass. Her medical history recorded a hysterectomy with bilateral ovariectomy 4 years before, with a diagnosis of uterine myxoid leiomyosarcoma (Fig. 1).

A neck ultrasound showed a $3.5-\mathrm{cm}$ nodule in the left lobe, confined to the thyroid, with no lymphadenopathy. The subsequent total body CT scan was unremarkable.

Fine-needle aspiration of the lesion demonstrated malignant, highly pleomorphic spindle cells, with many scattered giant cells (Fig. 2). The patient underwent a total thyroidectomy.

Grossly, the mass was well circumscribed and the cut surface was yellowish and hemorrhagic (Fig. 3). On histology, the lesion was well defined but unencapsulated (Fig. 4) and was composed by a proliferation of atypical spindle and epithelioid cells (Fig. 5, left), with a high mitotic rate, intermingled with many rather monomorphic giant cells (Fig. 5, right). The neoplastic spindle cells were set in a fibrotic stroma, with focal edematous areas. Immunohistochemical stains showed diffuse positivity for $\alpha$-smooth actin in the spindle cells (Fig. 6, upper panel) and for CD68/KP1 in the giant cells (Fig. 6, lower panel). All the other stains performed (desmin, S100 protein, cytokeratin
AE1/AE3, cytokeratin 7, cytokeratin 20, CD31, TTF1, thyreoglobulin, estrogen, and progesterone receptors) were negative. The neoplasia was confined within the thyroid capsule and no vascular invasion was noted. A diagnosis of possible metastatic leiomyosarcoma was rendered. The patient is alive and well without disease after an 18-months follow-up.

Among the differential diagnoses are anaplastic carcinoma (which we reasonably excluded based on the negativity of all the cytokeratin stains) and leiomyosarcoma, primitive, or metastatic. Thyroid leiomyosarcomas, either primitive $[1,2]$ or metastatic $[3,4]$, are extremely rare and show histologic characteristics superimposable to leiomyosarcomas arising in other sites.

In our case, the previous history is strongly in favor of a metastasis, but some important features are not: first of all, it is a solitary lesion (all the few cases reported in literature presented clinically with multiple metastasis at lungs and bones), moreover, it is completely different from its putative primary (see also Figs. 1 and 4) and the patient is well after 18 months.

So, can we admit that a patient develops two primary leiomyosarcomas at different sites? That's the question!

S. Piana $(\bowtie) \cdot$ R. Valli

Department of Pathology,

IRCCS-Arcispedale Santa Maria Nuova,

Viale Risorgimento 80,

42100 Reggio Emilia, Italy

e-mail: Simonetta.Piana@asmn.re.it

A. M. Foscolo

Department of Pathology, Ospedale Castelli Verbania,

Verbania, Italy 


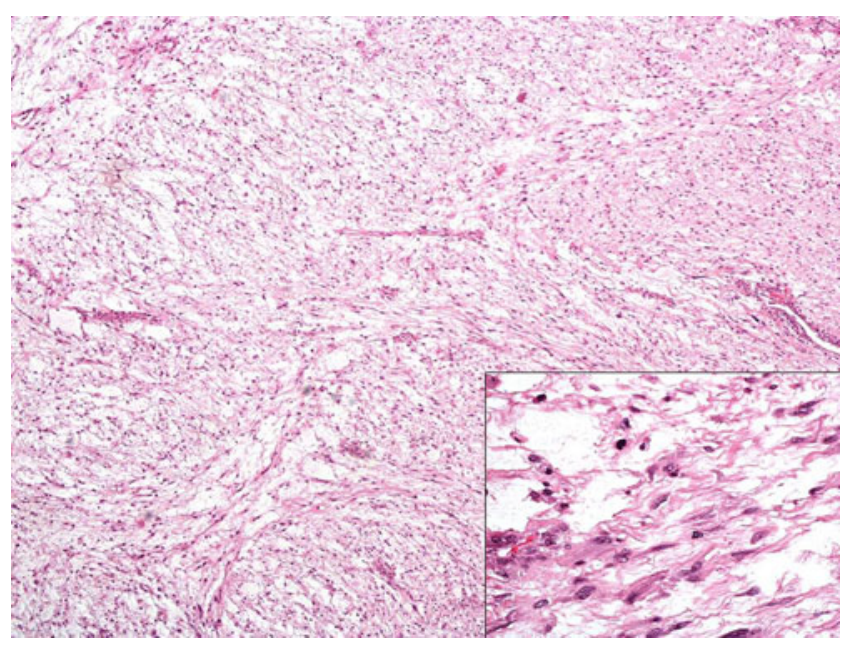

Fig. 1 Uterine leiomyosarcoma showed a diffusely myxoid stroma with atypical cells (inset)

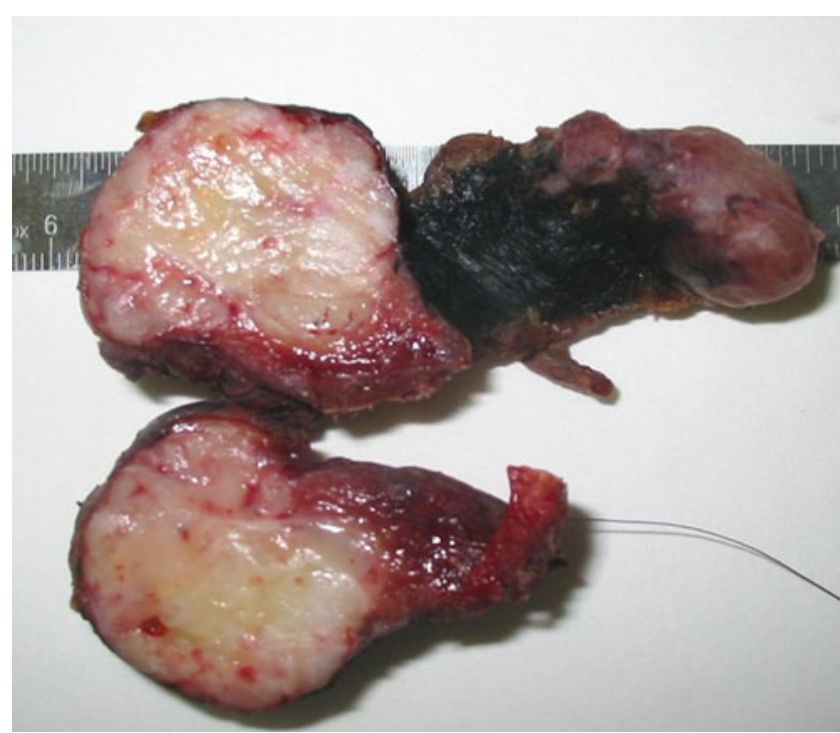

Fig. 3 Grossly, the mass was relatively well defined and limited to the thyroid

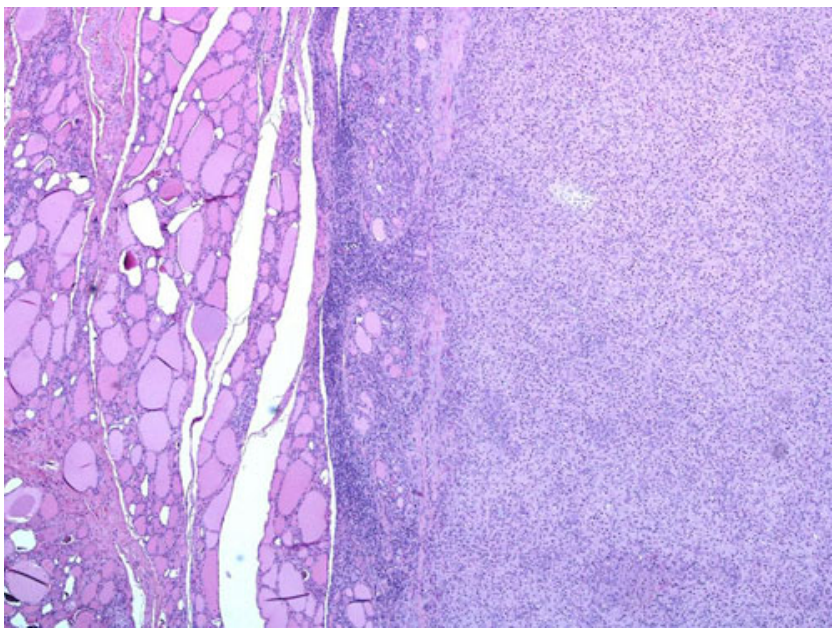

Fig. 4 At scanning magnification, the lesion was unencapsulated and highly cellulated

neoplastic spindle cells with some giant cells (arrows) 
Fig. 5 The tumor was composed by atypical spindle and epithelioid cells with a prominent mitotic activity (left) with many multinucleated giant cells scattered within the proliferating elements (right and inset)

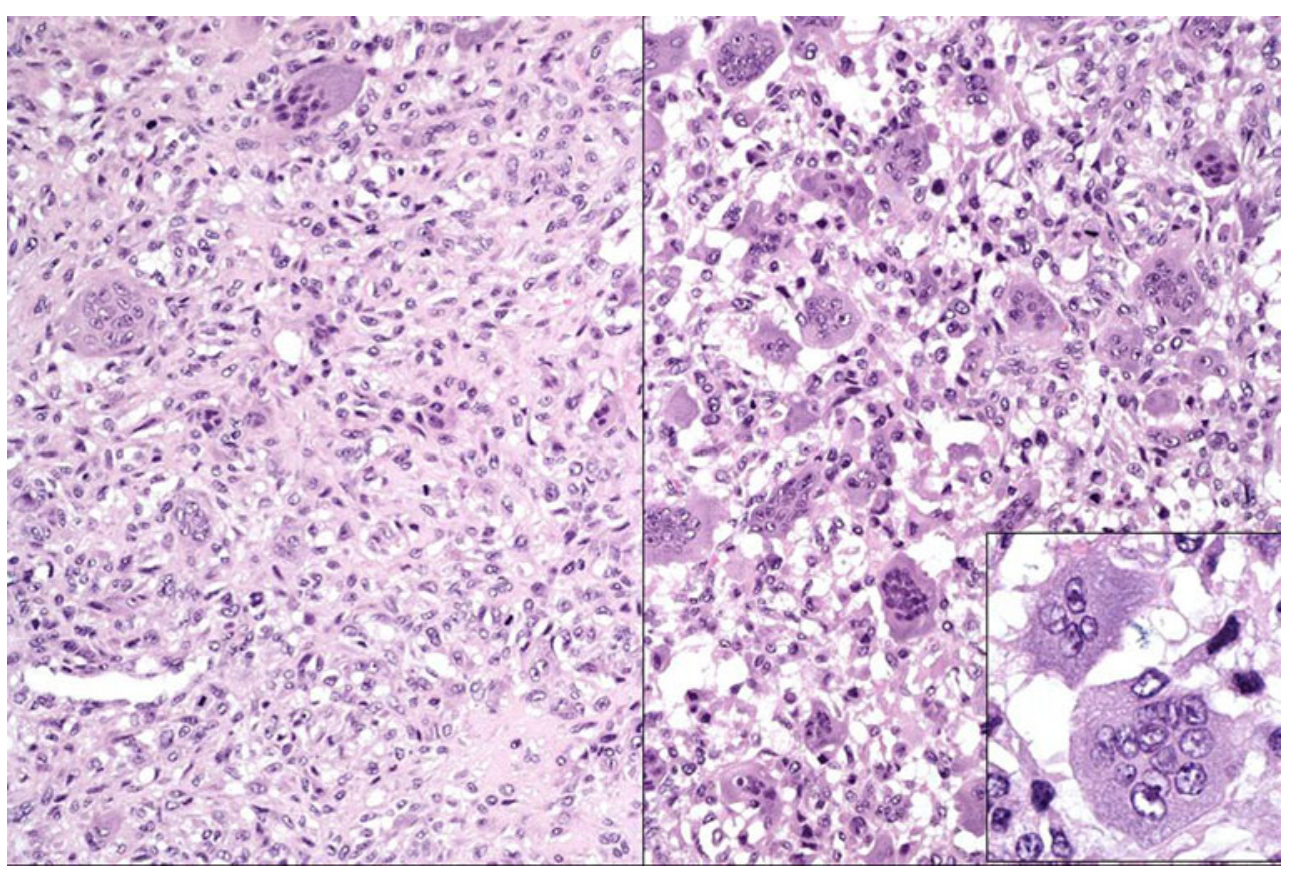

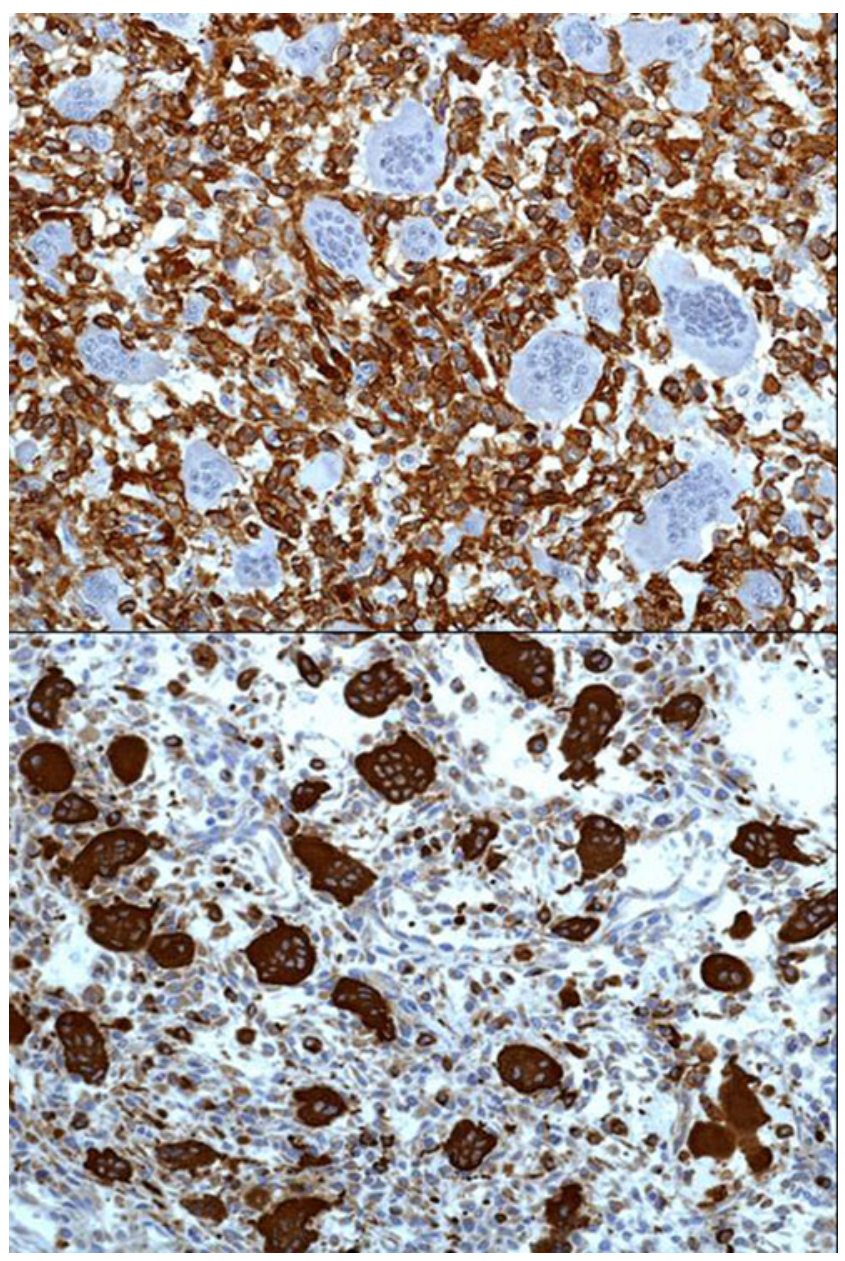

Fig. 6 At immunohistochemistry, $\alpha$-smooth muscle actin was intensely positive in the proliferating cells (top) but negative in the multinucleated elements, while CD68 was negative in the former but stained diffusely the latter (bottom)

\section{References}

1. Wang TS, Ocal IT, Oxley K, Sosa JA. Primary leiomyosarcoma of the thyroid gland. Thyroid 18:425-428, 2008

2. Mansouri H, Gaye M, Errihani H, Kettani F, Gueddari BE. Leiomyosarcoma of the thyroid gland. Acta Otolaryngol 128:335336, 2008

3. Leath CA, Huh WK, Straughn JM, Conner MG. Uterine leiomyosarcoma metastatic to the thyroid. Obstet Gynecol 100:1122-1124, 2002

4. Anderson Eloy J, Mortensen M, Gupta S, Lewis MS, Brett EM, Genden EM. Metastasis of uterine leiomyosarcoma to the thyroid gland: case report and review of the literature. Thyroid 17; 1295 1297, 2007 